\title{
Collaborative Positioning Mechanism Using Bayesian Probabilistic Models for Industry Verticals
}

\author{
Carlos H. M. de Lima, Jani Saloranta and Matti Latva-aho \\ Centre for Wireless Communications, University of Oulu, Oulu, Finland \\ E-mail: \{carlos.lima, jani.saloranta, matti.latva-aho\}@oulu.fi
}

\begin{abstract}
In this paper, we develop a collaborative positioning mechanism which uses Bayesian probabilistic models to combine multidimensional sensory data and localize target nodes over the network deployment area. Herein, heterogeneous anchor nodes with distinct radio access technologies and experiencing various radio channel features implement a joint sensor fusion and positioning system for industry verticals. The proposed mechanism also relies on a modern network architecture whereby devices offload high-demand computation to more capable edge servers which then estimate the target node position after gathering anchors measurements and prior history. Kernel density estimation results are used to show that edge servers implementing Bayesian-based sensor fusion and positioning system effectively estimate the target node location when using hybrid metrics and combining past and current sensory inputs.
\end{abstract}

\section{INTRODUCTION}

The next generation of wireless communications will not be a straightforward evolution of current systems, but instead it will disrupt traditional design principles and revolutionize the way we live, interact and make business. Legacy networks have been always designed as general purpose connecting platforms with very limited variation of functionalities across deployment scenarios and use cases. On the other hand, the upcoming 5G New Radio (NR) will cope with specific requirements from onset so as to integrate digital technologies into vertical markets as well as our every day life. In fact, the next generation will merge the physical and digital worlds and by doing so realize the concept of a true networked society [1]. To achieve that, four key technologies are converging: 5G and beyond cellular systems, Artificial Intelligence (AI), Data analytic and Internet of Things (IoT). In fact, pundits advocate this ongoing convergence process will revolutionize the Information and Communications Technology (ICT) ecosystem and drastically alter all major industry verticals [2].

$5 \mathrm{G}$ NR will become a catalyst for this revolution by bringing about new unique set of features and service capabilities: i) enhanced broadband communication providing seamless experience even at high mobility; $i i$ ) massive deployment of smart objects (internet of things); and iii) mission critical services requiring very high reliability and/or very low latency. Thus, it will allow for close inter-working between the ICT industry and vertical sectors players and then enable a wide range of innovative use cases. Equally important, such new services will require advanced enablers such as Sensing, imaging, environmental monitoring, security, trust, and particularly accurate positioning [3].
Indeed, positioning is a key enabler for the new services in the upcoming wireless communications, for example, transportation, logistics, health care, animal husbandry, environmental monitoring and even wearables [4]-[6]. Our solution assumes a modern network infrastructure whereby nodes offload their computation to Multi-Access Edge Computing (MEC) servers that estimate the target node position in a centralized manner. In this configuration, less capable Location Measurement Unit (LMU) are connected to the edge servers and forward measurements accordingly. With this distributed configuration, any previous knowledge about the network disposition is available and then used to update (or improve) the "believe" about the target location. Many industry vertical applications rely on low capacity devices (either communication or computation), but require or benefit from location, thus the proposed MEC configuration allow for fast deployment of new applications and timely execution of highly demanding tasks [7]. In the scenarios under consideration, access (anchors) points, supporting localization units and low computation power terminals offload their computation to nearby interconnected edge servers that estimate the target node position fusing measurements from heterogeneous sources throughout the proposed Bayesian graphical model.

In this contribution, probabilistic graphical models (Bayesian Networks), whose underlying structure is represented by Directed Acyclic Graphs (DAGs), are used to model and solve localization problems. The driving idea is to develop a robust mechanism by which current measurements and previous knowledge (sequential information) are combined so as to improve overall accuracy while guaranteeing communication transactions. In [5], Madigan et al. introduced a Bayesian hierarchical model to carry out indoor localization estimation using Markov Chain Monte Carlo (MCMC) and Gibbs sampler. They also highlighted the relevance of the zero profiling concept which uses prior knowledge about the nodes disposition to eliminate the need for training data. Along the same lines, Authors in [8] develop hybrid localization solutions by combining Received Signal Strength (RSS) and Time of Arrival (TOA) in a common framework that is more robust to variable measurement precision. Equally important, the device heterogeneity of modern applications (transmitter and receiver with conditionally-related parameters) is addressed in [9] using a hierarchical Bayesian probabilistic model. 
Herein, we also consider such Bayesian formulation to estimate the position of the target node and use that as service enabler for future network deployments that benefit from higher computational power of the MEC servers [10].

Our main contributions are summarized as follows:

- A Bayesian formulation for positioning in indoor Machine Type Communication (MTC) deployments wherein nodes have limited computational and depend on edge servers to carry out the localization procedures.

- Different from previous works, the Hamiltonian Monte Carlo algorithm is used to sample the posterior distributions of the target node coordinates.

The reminder of this paper is organized as follows. Section III presents the channel propagation and network deployment models, as well as establishes the evaluation scenario under investigation. Afterwards, we introduce the collaborative localization framework based on Bayesian probabilistic models in Section II, while discussing its implementation details and pointing out (dis)advantages of the proposed formulation. In Section IV, we carry out extensive simulation campaign using the MCMC approach so as to assess the performance of the Bayesian-based framework in terms of the Kernel Density Estimation (KDE) and Root Mean Square Error (RMSE) for distinct configuration parameters and decision metrics, namely RSS, TOA and combinations thereof. Conclusions and final remarks are drawn in Section V.

\section{Probabilistic Graphical Models}

Bayesian graphical models allow to pictorially represent a joint distribution $f\left(x_{1}, x_{2}, \ldots, x_{n}\right)$ relating a set of random variables $\left\{X_{1}, X_{2}, \ldots, X_{n}\right\}$ throughout the conditional independence assumptions underlying the statistical model. As Authors in [11] point out, a DAG representation provides a general-purpose modeling language for exploiting this type of probabilistic structure. The relationships between Random Variables (RVs) are then given by a DAG $\mathcal{G}=(V, E)$ with $V$ vertices and $E$ edges representing RVs and their corresponding inter-dependencies, respectively. Let $X=\left(X_{V}\right)$ be a set of RVs indexed by $V$. From [11], $X$ represents a Bayesian Network with respect to $\mathcal{G}$ if its joint probability density function (with respect to a product measure) can be written as a product of the individual density functions, conditional on their parent variables,

$$
f(V)=\prod_{v \in V} f(v \mid \mathrm{pa}[v])
$$

where $\mathrm{pa}[v]$ is the set of parents nodes of $v$ (i.e. those vertices pointing directly to $v$ via a single edge). As highlighted by Spiegelhalter in [12], by simply interpreting RVs as vertices and representing their intrinsic statistical relations through the graph edges, one can connect the underlying DAG and Bayesian networks as given in (1) - any node is conditionally independent of all non-descendants given the parents.
As a result, the conditional distribution for any such vertex (representing a random variate) $v \in \mathcal{G}$ is given by,

$$
\begin{aligned}
f(v \mid V \backslash v) & \propto f(v, V \backslash v) \\
& \propto \text { terms in } f(V) \text { containing } v \\
& =f(v \mid \operatorname{pa}[v]) \prod_{w \in \operatorname{ch}[v]} f(w \mid \operatorname{pa}[w]),
\end{aligned}
$$

where $\operatorname{ch}[v]$ yields the set of children of the node $v$.

Thus, DAGs are employed here to capture the qualitative relationships between the random quantities. Aiming to obtain valid posterior estimates for the unknown variates of interest, we first need to specify an suitable statistical model describing the interdependence between the relevant parameters. Thereafter, MCMC method (a powerful generic computational approach) is employed to approximately sample from arbitrary distributions [13]. We resort to Bayesian probabilistic models to develop our collaborative localization procedure [11], [14]. In particular, the Hamiltonian Monte Carlo approach is employed to carry out the MCMC method in a computationally efficient manner - which is elegantly formalized throughout the Hamiltonian dynamics [15], [16]. Succinctly, MCMC method is a generic approach for sampling from an arbitrary distribution. The main idea is to generate a Markov chain whose limiting distribution is equal to the desired distribution [17]

\section{EVALUATION SCENARIO AND LOCALIZATION MECHANISM}

In this section, we initially detail the propagation and deployment models used to build the evaluation scenario, and thereafter describe our Bayesian-based collaborative positioning system - which combines current and past measurements to accurately estimate the target node location.

\section{A. Channel Propagation Model}

The radio links between communicating nodes are degraded by a log-distance shadowed path loss model which is assumed to be independent over distinct network entities and positions. Thus, the RSS follows a decay function given by,

$$
\rho_{i}=\rho_{0}-\eta \log \left(D_{i}\right)+\chi,
$$

where $\rho_{i}$ is the RSS at the target node relative to the $i$ th anchor, $\rho_{0}$ is the received power at an arbitrary reference distance (assumed to be $1 \mathrm{~m}$ ), $\eta$ is the path loss exponent, $\chi$ is shadowing with zero mean normal distribution and standard deviation $\sigma_{i}$ in the logarithmic scale, and $D_{i}$ is the Euclidean distance between the $i$ th anchor and the target node given by,

$$
D_{i}=\sqrt{\left(X-x_{i}\right)^{2}+\left(Y-y_{i}\right)^{2}},
$$

where $(X, Y)$ is a bidimensional variate representing the target node position, while $\left(x_{i}, y_{i}\right)$ corresponds to the predefined $i$ th anchor node location. 


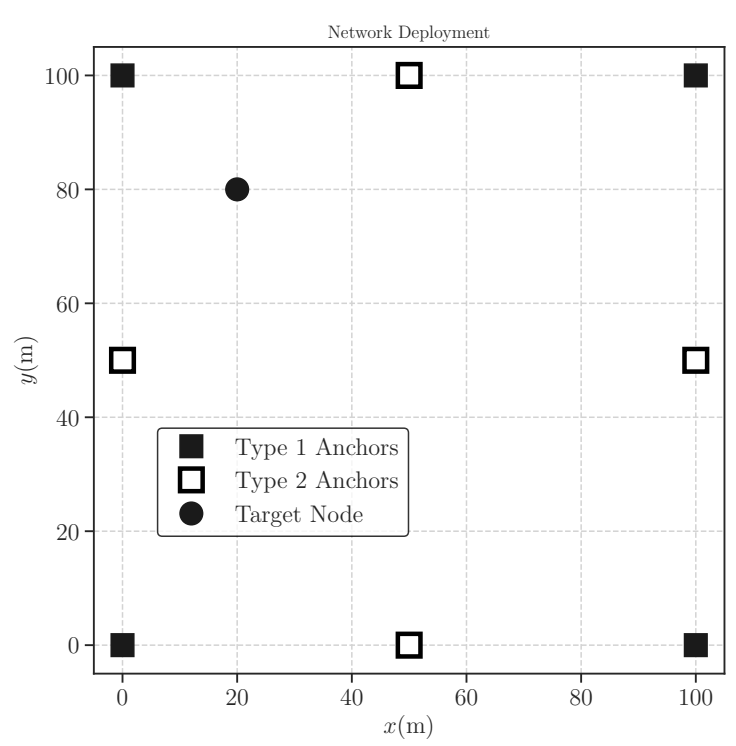

Fig. 1: Illustration of the network deployment model. Filled and hollowed squares represent type 1 and 2 anchor nodes, respectively, while the circle at $(20,80) \mathrm{m}$ represents the actual target position.

\section{B. Network Deployment Model}

Fig. 1 illustrates the evaluation scenario with dimensions $L$ and $W$. Without lack of generality, short internode distances and predominantly Line-of-Sight (LoS) operation are assumed. From this figure, $n$ reference nodes at known positions $\left(x_{i}, y_{i}\right)$ collaborate to locate a target node with arbitrary position $(X, Y)$. Following our problem formulation, each such anchor node independently gets measurements from the target with the purpose of estimating its location. Two types of anchor nodes (regarding how target node measurements are acquired) are considered throughout these investigations: filled squares are more capable Access Points (APs) with more advanced radio access technology which, in addition to RSS measurements, are also capable of collecting more elaborated metrics such as TOA and Direction of Arrival (DOA). Differently, hollowed squares are simpler LMU that can acquire just one positioning metric type. The positioning solution is evaluated considering two distinct test scenarios: i) a squared warehouse with four anchors (known locations) positioned at the respective corners collecting one metric only (either RSS or TOA); and ii) anchors collecting two distinct metrics in a hybrid solution.

\section{Localization mechanism}

The proposed collaborative positioning systems accounts for the distributed nature of scenarios under investigation and the corresponding heterogeneity of the communicating devices. An edge-based approach whereby current and past measurements from all localization agents are aggregated and then used to localize the target node [18]. Next, we make use of the DAG representation to formulate the localization problem as a Bayesian probabilistic model.
In the following we present the plate notation (which shows the conditional interdependence between RVs) and respective formulation for the metrics considered here.

1) RSS-based localization mechanism: Fig. 2 presents the plate notation [5], [19] for the RSS-based mechanism described in (5). In this diagram, circular vertices represent $\mathrm{RVs}$, arrow edges represent dependencies between RVs and symbols inside rectangles correspond to constant values. The underlying assumptions and respective prior distributions used to build the model and sample the posterior distribution are given in (5). Similarly, Section III-C2 shows the TOA-based probabilistic graphical models.

The specification of the DAG model vertices conditional densities given their corresponding parent nodes.

$$
\begin{aligned}
X & \sim \operatorname{Uniform}(0, L), \\
Y & \sim \operatorname{Uniform}(0, W), \\
D_{i} & \sim \sqrt{\left(X-x_{i}\right)^{2}+\left(Y-y_{i}\right)^{2}}, \\
\mu_{i} & \sim \rho_{0 i}+\eta_{i} \log \left(D_{i}\right), \\
\rho_{0 i} & \sim \operatorname{Normal}(0,100), \\
\eta_{i} & \sim \operatorname{Normal}(0,100), \\
\sigma_{i}^{2} & \sim \operatorname{HalfNormal}(10),
\end{aligned}
$$

where $(X, Y)$ is a bidimensional variate representing the target node position, $D_{i}$ is the separation distance between the target node and the $i$ th anchor, $\rho_{0 i}$ is the power received at an arbitrary reference position (assumed to be $1 \mathrm{~m}$ ), $\eta_{i}$ is the path loss exponent and $\sigma_{i}$ is the corresponding standard deviation associated to the $i$ th anchor measurements.

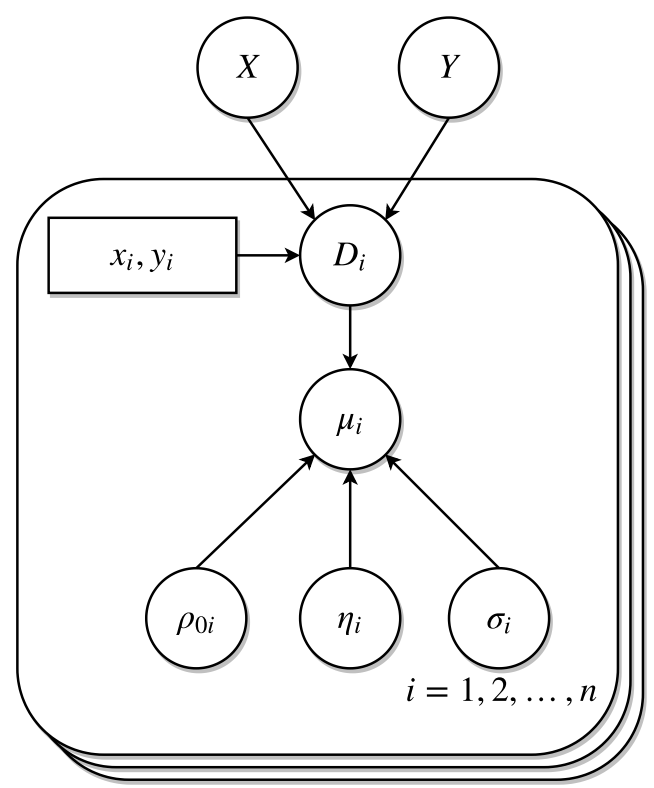

Fig. 2: Illustrative Bayesian probabilistic model using plate notation for the collaborative RSS-based localization mechanism. 


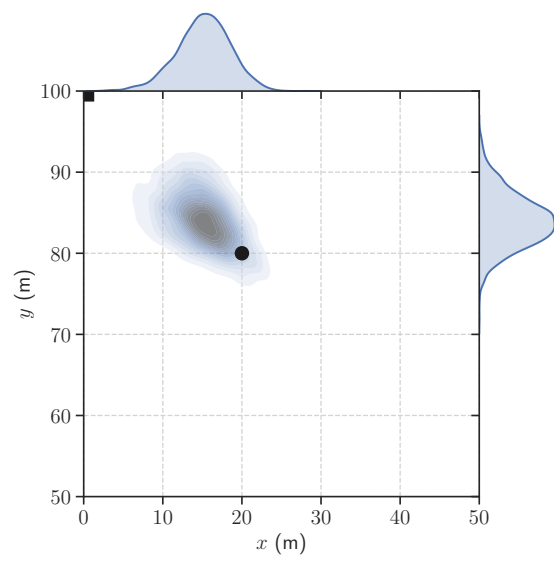

(a)

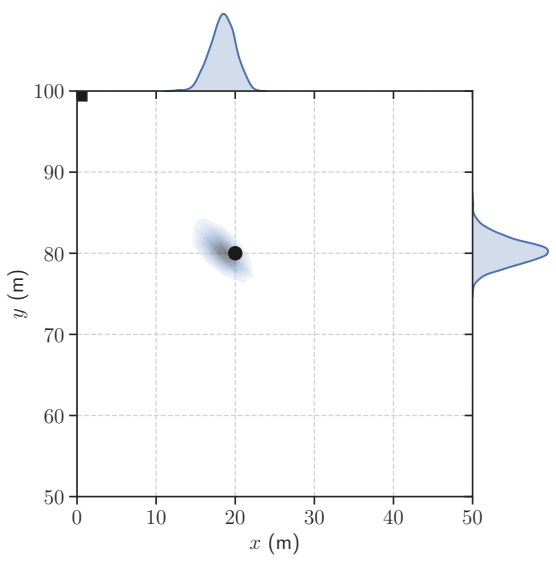

(b)

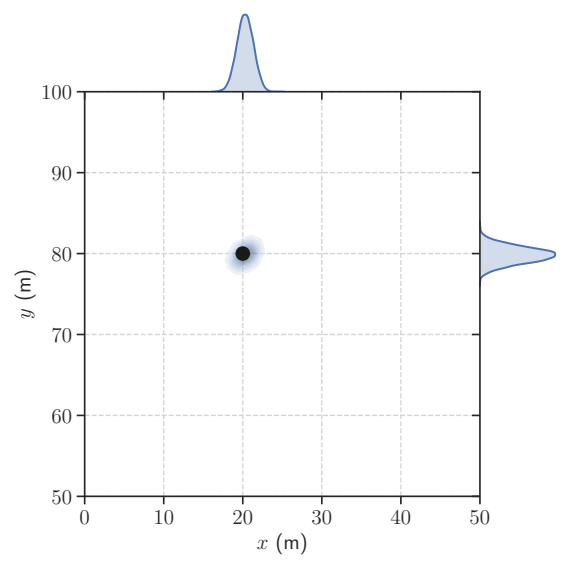

(c)

Fig. 3: KDE for the positioning system employing (a) 50 and (b) 150 RSS measurements, (c) 50 TOA measurements. The black dot represents the actual target position at $(20,80) \mathrm{m}$

2) TOA-based localization procedure: Similarly, the TOA-based Bayesian network is described in (6).

$$
\begin{aligned}
X & \sim \operatorname{Uniform}(0, L), \\
Y & \sim \operatorname{Uniform}(0, W), \\
D_{i} & \sim \sqrt{\left(X-x_{i}\right)^{2}+\left(Y-y_{i}\right)^{2}}, \\
\mu_{i} & \sim \frac{D_{i}}{c}, \\
\sigma_{i}^{2} & \sim \operatorname{HalfNormal}(10),
\end{aligned}
$$

where $c$ corresponds to the speed of light.

In these investigations, we also consider hybrid solutions by combining distinct metrics, namely RSS and TOA into the Bayesian network framework. Aiming at saving space, the DAG hybrid approach is not explicitly shown, though it merges elements from (5) and (6) constructions.

\section{Performance AnAlysis}

In this section, we carry out an extensive simulation campaign to assess the collaborative Bayesian-based positioning system using the KDE of the posterior distribution of the target location coordinates and the respective RMSE. Monte Carlo simulations are used to generate measurements according to the propagation and deployment models from Section III. The Hamiltonian Monte Carlo algorithm [20] is then used to sample the posterior distribution of the bidimensional RV representing the target position $(X, Y)$.

Fig. 3 summarizes the KDE for the collaborative positioning system using distinct metrics, namely RSS and TOA, as well as variable number of measurements per anchor node. As can be seen from Figs. 3(a) and (b), the RSS is inherently less accurate and needs much more valid measurements to provide reasonable positioning estimates. On the other hand, the TOA-based approach provides comparable results by using much less measurements as shown in 3(c).
When combing RSS measurements from distinct anchors at the edge server, Fig. 4 presents the violin plot for the $x$ coordinate of the target node position and respective RMSE. The RSS metric is more susceptible to channel impairments and intrinsically less precise thus requiring more measurements to provide reasonable estimates. The collaborative mechanism provides increasingly better estimates with the number of measurements (which can be combined at the Bayesian module even if they are not collected simultaneously). The measurements are collected by anchors located at the corners of the test scenario shown in 1 . The number of measurements are shown in the vertical axis, while the $x$ coordinate estimate is shown in the horizontal one.

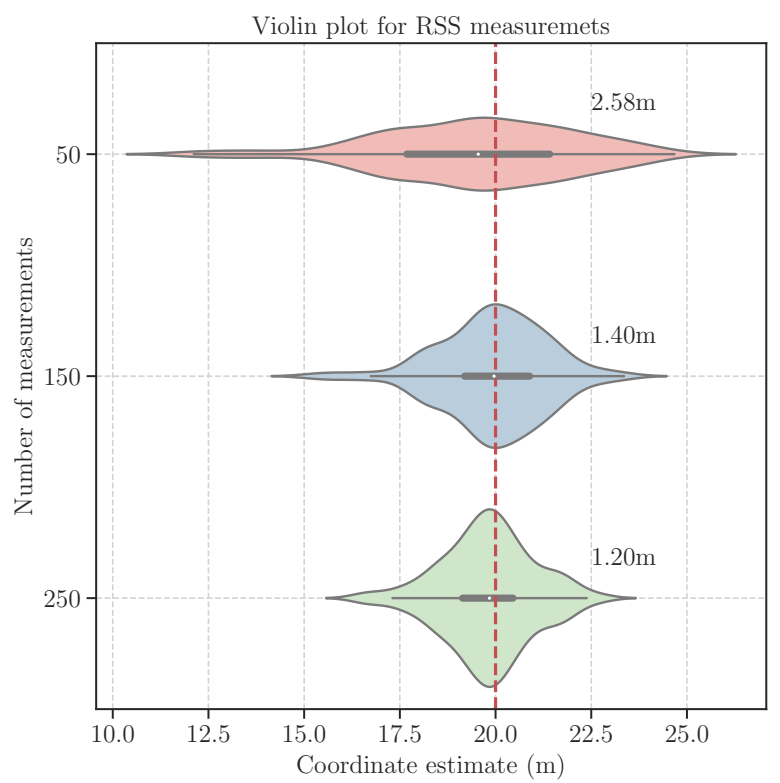

Fig. 4: Violin plot and RMSE for the collaborative positioning system using RSS measurements only. 


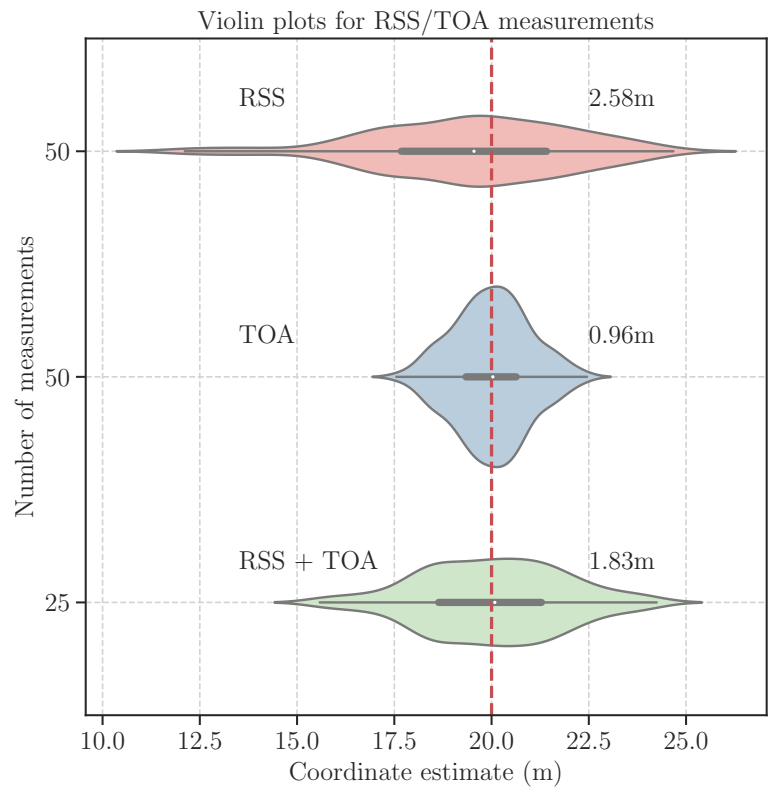

Fig. 5: Violin plot and RMSE for the collaborative positioning system using RSS and TOA measurements.

Similarly, Fig. 5 compares the resulting posterior distributions for distinct metrics and combinations thereof. It is worth mentioning that the collaborative localization system does not need to assume beforehand the availability of any specific metric, but instead makes use of the available measurements as they become available. Here, RSS- and TOA-based positioning framework collect measurements from all anchors located at the corners of the test scenario. On the other hand, the RSS- plus TOA-based approach collects signal strength from the bottom anchors, and time of flight measurements from the top anchors as illustrate in Fig. 1. In fact, by combining both metrics, namely signal strength and time of flight, the RSS- plus TOA-based approach need less measurements so as to provide comparable results. In this test scenario, each anchor node collects and forwards 25 measurements of the respective metrics. It is also worth mentioning that this framework allows combining heterogeneous devices with distinct radio access technologies into a common localization framework.

\section{CONCLUSIONS}

Herein, we assess the feasibility of Bayesian networks to combine current and past measurements from distinct anchors at edge servers. Then, the MCMC method is used to compute the Bayesian statistics and evaluate how the collaborative localization solution perform in the heterogeneous deployment scenarios. Particularly, the Hamiltonian Monte Carlo algorithm is used to sample from the posterior distribution and estimate the target node position. Our results show that Bayesian probabilistic models can effectively combine hybrid positioning metrics from various heterogeneous sources with distinct radio access technologies and computational capabilities.

\section{ACKNOWLEDGMENT}

The research leading to these results has received funding from the Academy of Finland through the projects 6Genesis Flagship (Grant No. 318927), and Positioning-aided Reliably-connected Industrial Systems with Mobile mmWave Access (PRISMA).

\section{REFERENCES}

[1] 5GPP, "White paper: The 5G infrastructure public private partnership: the next generation of communication networks and services," acessado: July 3, 2019

[2] G. A. Akpakwu, B. J. Silva, G. P. Hancke, and A. M. Abu-Mahfouz, "A survey on $5 \mathrm{G}$ networks for the internet of things: Communication technologies and challenges," IEEE Access, vol. 6, pp. 3619-3647, 2018

[3] X. Lin, J. Bergman, F. Gunnarsson, O. Liberg, S. M. Razavi, H. S. Razaghi, H. Rydn, and Y. Sui, "Positioning for the internet of things: A 3gpp perspective," IEEE Communications Magazine, vol. 55, no. 12, pp. 179-185, 2017.

[4] R. Di Taranto, S. Muppirisetty, R. Raulefs, D. Slock, T. Svensson, and $\mathrm{H}$. Wymeersch, "Location-aware communications for $5 \mathrm{~g}$ networks: How location information can improve scalability, latency, and robustness of 5g," IEEE Signal Processing Magazine, vol. 31, no. 6, pp. 102-112, 2014.

[5] D. Madigan, E. Einahrawy, R. P. Martin, W.-H. Ju, P. Krishnan, and A. Krishnakumar, "Bayesian indoor positioning systems," in Proceedings IEEE 24th Annual Joint Conference of the IEEE Computer and Communications Societies., vol. 2. IEEE, 2005, pp. 1217-1227.

[6] A. Nguyen, N. Milosavljevic, Q. Fang, J. Gao, and L. J. Guibas, "Landmark selection and greedy landmark-descent routing for sensor networks," in 26th Annual Joint Conference of the IEEE Computer and Communications Societies, 2007. INFOCOM 2007. IEEE, May 2007. pp. 661-669.

[7] E. S. Lohan, M. Koivisto, O. Galinina, S. Andreev, A. Tolli, G. Destino, M. Costa, K. Leppanen, Y. Koucheryavy, and M. Valkama, "Benefits of positioning-aided communication technology in high-frequency industrial iot," IEEE Communications Magazine, vol. 56, no. 12, pp. 142-148, 2018.

[8] R. Kim, H. Lim, S.-N. Hwang, and B. O. Obele, "Robust indoor localization based on hybrid bayesian graphical models," in 2014 IEEE Global Communications Conference. IEEE, 2014, pp. 423-429.

[9] K. F. Davies, I. G. Jones, and J. L. Shapiro, "A bayesian approach to dealing with device heterogeneity in an indoor positioning system," in 2018 International Conference on Indoor Positioning and Indoor Navigation (IPIN). IEEE, 2018, pp. 1-8.

[10] Y. Mao, C. You, J. Zhang, K. Huang, and K. B. Letaief, "A survey on mobile edge computing: The communication perspective," IEEE Communications Surveys Tutorials, vol. 19, no. 4, pp. 2322-2358, Fourthquarter 2017.

[11] D. Koller, N. Friedman, and F. Bach, Probabilistic graphical models: principles and techniques. MIT press, 2009.

[12] D. J. Spiegelhalter, "Bayesian graphical modelling: a case-study in monitoring health outcomes," Journal of the Royal Statistical Society: Series C (Applied Statistics), vol. 47, no. 1, pp. 115-133, 1998.

[13] D. Gamerman and H. F. Lopes, Markov chain Monte Carlo: stochastic simulation for Bayesian inference. Chapman and Hall/CRC, 2006.

[14] K. P. Murphy, "Machine learning: A probabilistic perspective (adaptive computation and machine learning series)," 2018.

[15] A. Gelman, H. S. Stern, J. B. Carlin, D. B. Dunson, A. Vehtari, and D. B. Rubin, Bayesian data analysis. Chapman and Hall/CRC, 2013.

[16] S. Brooks, A. Gelman, G. Jones, and X.-L. Meng, Handbook of markov chain monte carlo. CRC press, 2011.

[17] D. P. Kroese, T. Taimre, and Z. I. Botev, Handbook of monte carlo methods. John Wiley \& Sons, 2013, vol. 706.

[18] H. Wymeersch, J. Lien, and M. Z. Win, "Cooperative localization in wireless networks," Proceedings of the IEEE, vol. 97, no. 2, pp. 427-450, 2009.

[19] W. L. Buntine, "Operations for learning with graphical models," Journal of artificial intelligence research, vol. 2, pp. 159-225, 1994.

[20] J. Salvatier, T. V. Wiecki, and C. Fonnesbeck, "Probabilistic programming in python using pymc3," PeerJ Computer Science, vol. 2, p. e55, Apr. 2016. [Online]. Available: https://doi.org/10.7717/peerj-cs.55 